\title{
Reply: The Characteristics about Our Study in Irritable Bowel Syndrome
}

\author{
Kang-Min Zhuang, Yu-Bin Guo, and Si-De Liu \\ Guangdong Provincial Key Laboratory of Gastroenterology, Department of Gastroenterology, Nanfang Hospital, Southern Medical University, \\ Guangzhou, China
}

\section{Reply:}

At the time of publication, very few population-based studies had investigated the roles of diet and lifestyle habits in irritable bowel syndrome (IBS) in developing countries, particularly in China. ${ }^{1}$ The aim of this preliminary study was to investigate the association between diet and lifestyle habits and IBS. Thus, we investigated information prior to the first occurrence of the syndrome, excluding the other direction, such that IBS may have been the cause of specific diet and lifestyle habits. Our workplace was a large-scale general hospital in southern China. The patients admitted to the hospital were from all regions of the country. Thus, the patients enrolled in our study originated from different parts of the country with different socioeconomic statuses or different types of environmental pollution. Furthermore, in the design of this study, the first principle was to set strict criteria for the enrollment of patients who both fulfilled the diagnosis of IBS and also had a relatively clear memory regarding changes in clinical symptoms. These criteria were clearly described in our paper. Although this approach significantly excluded a substantial number of patients who may have been IBS patients, it also improved the overall quality of our study. Furthermore, there had also been an IBS study with few but high quality samples. ${ }^{2}$ We did not discuss the limitations of the conclusion drawn from the present study; however, it is gener- ally accepted that the level of evidence regarding case control studies is relatively low according to the grading system of evidence-based medicine. ${ }^{3}$ Thus, it is more appropriate to regard our research as a reference for further research, such as cohort studies or randomized clinical trials, rather than an unalterable conclusion.

\section{CONFLICTS OF INTEREST}

No potential conflict of interest relevant to this article was reported.

\section{REFERENCES}

1. Guo YB, Zhuang KM, Kuang L, Zhan Q, Wang XF, Liu SD. Association between diet and lifestyle habits and irritable bowel syndrome: a case-control study. Gut Liver 2015;9:649-656.

2. Su YC, Wang WM, Wang SY, et al. The association between Helicobacter pylori infection and functional dyspepsia in patients with irritable bowel syndrome. Am J Gastroenterol 2000;95:1900-1905.

3. Grondin SC, Schieman C. Evidence-based medicine: levels of evidence and evaluation systems. In: Ferguson MK, ed. Difficult decisions in thoracic surgery. London: Springer London, 2011:13-22.

Correspondence to: Si-De Liu

Guangdong Provincial Key Laboratory of Gastroenterology, Department of Gastroenterology, Nanfang Hospital, Southern Medical University, Guangzhou, Guangdong Province, China

Tel: +86-13265036054, Fax: +86-2061641114, E-mail: liuside2013@163.com.

Received on July 13, 2016. Accepted on July 19, 2016. Published online November 24, 2016

pISSN 1976-2283 eISSN 2005-1212 https://doi.org/10.5009/gnl16346

(a) This is an Open Access article distributed under the terms of the Creative Commons Attribution Non-Commercial License (http://creativecommons.org/licenses/by-nc/4.0) which permits unrestricted non-commercial use, distribution, and reproduction in any medium, provided the original work is properly cited. 